\title{
Dismantling the online sphere - Information-filters as organizers of public vision
}

\author{
Anders Koed Madsen PhD Fellow, Department of Organization, Copenhagen Business School
}

This paper shows how searches for "synthetic biology" on Google.co.uk and Wikipedia.com lead to different demarcations of this controversy in the form of the hyperlink networks that become browsable for the user. When talking about the mediation of biotechnological controversies in the public sphere the interesting divide may accordingly not be between the offline and the online, but rather between logics of filtering on the web and the networks of actors that are playing a role in assembling the "web-visions" they give rise to.

\section{1: Introduction}

Debates about whether the Internet provides a valuable public sphere have been reoccurring since the mid-90s and they have often centered around differences and similarities between the so-called "online public sphere" and its offline counterpart. The methodological trend in such studies has been to carry out representative samples of the discussion about a given topic in each of the two spheres and use these samples as a basis for discussing whether the online sphere makes room for a more or less direct (Coleman 2005), plural (Gerhards, Schaefer 2010), deliberative (Marlin-Bennett 2011), de-centralized (Benkler 2006, Surowiecki 2004), epistemic correct (Goldman 2008), participatory (O'Reilly 2006) or representative (Papacharissi 2002, Lazer et al. 2009) sphere than the one designated as offline. Scholars behind these studies have disagreed on the democratic consequences of the Internet but they have nonetheless agreed on approaching it as an online sphere that can be more or less representatively uncov- ered by the researcher (Lazer et al. 2009, Schneider, Foot 2005, Thelwall 2009).

Section 3 of this paper outlines the contours of this debate and indicates that the online/offline divide may not be the most fruitful framework for understanding the web as a public arena. It will be argued that conceptualizations that posit the online sphere as a unified phenomenon capable of being compared to other spheres are increasingly challenged by the role which informationfilters and their surrounding networks play in organizing the visibility of voices around specific issues (Halavais 2009). More specifically the section uses the concept of "web-visions" (Koed Madsen, 2012) as a way to denote the slice of a controversy that is made visible by an information-filter to a temporally and spatially situated web-user. The concept is operationalized by following hyperlinks with a departure in the concrete scope of information returned by specific filters of interest. A "web-vision" is therefore not representative of anything outside the filter but it can provide an understanding of the way computers, web-masters, algorithms and web-users play a role in demarcating controversies and how this demarcation differs in relation to the used filter that serves as the entry-point to the web as well as the time and place of the entry.

The empirical part of the paper is an analysis of the way the controversy of synthetic biology has been organized and demarcated by two of the most heavily used information-filters in the UK, wikipedia.com and google.co.uk, from January - June 2011. Synthetic biology is the most recent attempt at applying planned engineering on living organisms and section 2 gives an introduction to the choice of this case and the choice 
of looking at the UK and it also outlines some general thoughts about the democratic importance of sociotechnical controversies. The data presented in the paper represents the beginning of a longer longitudinal design and it should therefore be read as an explorative attempt at using the dynamics of these situated "web-visions" to conceptualize the relation between the web and sociotechnical controversies in a way that is different than working from the basis of samples of web-sites as more or less accurate representations of an online sphere.

Section 4 contains the results of the initial quantitative analysis of the web-visions and it shows how google. co.uk gives rise to more fluid demarcations of the issues of synthetic biology than wikipedia.com and how e.g. artists are visible as voicing social and ethical questions about the technology in the Google-vision. This stands in contrast to the more stable and institutionalized demarcation emerging from wikipedia.com but the longitudinal study also shows how the web-visions get more and more similar over time. Section 5 provides a discussion of the findings of the analysis and presents three arguments for the usefulness of working with web-visions instead of starting from pre-defined spheres. Section 6 concludes the paper and points towards possibilities for further research.

\section{2: Synthetic biology as a socio-technical controversy}

Scholars within the tradition of Science and Technology Studies (STS) have since the 1970s carried out a range of studies showing different ways in which the development of science is always intermeshed with politics in a way that poses hard questions about its role in a democratic society. A few highlights of this literature have been studies illustrating how the development of nuclear radioactivity carried with them discussions about the divide between experts and laymen in decision-making (Wynne, 1992), how the meaning of technology assessment in relation to GMO have to a large extent been influenced by local cultures and traditions of law-making (Jasanoff, 2007) and how knowledge-claims in the realm of science are often settled in coalitions of a diverse set of human actors in combination with non-human actors such as laws and scientific equipment (Latour, 1987). Irwin and Michael have coined the concept of ethno-epistemic assemblages in order to more precisely express the idea that such "hybrid coalitions" put forward knowledgeclaims in relation to specific socio-scientific controversies (epistemic) in a way that is situated in local contexts where it is possible to trace back knowledge-claims to local practices (ethno). By calling these ethno-epistemic coalitions assemblages, Irwin and Michael emphasize that they connote a territory of heterogeneous actors that form a whole through their relations to each other and the signs that enable communication between them (Irwin \& Michael, 2003). The web, which we will return to in the next section, is without doubt an important actor in establishing such signs and relations today.

With the insights from 30 years of STS research in mind it is not surprising that synthetic biology has enrolled a range of different actors in discussions about its development. As the most recent example of genetic engineering of biological organisms follows a list of scientific projects that have previously created controversies by framing themselves as providers of answers to large societal questions such as food hunger, climate change and disease prevention. Since the first organisms were genetically engineered in the 1970s there have been immense political and ethical debates between proponents of these biotechnological solutions to societal problems and critics who have deemed such solutions to be unsafe, unethical or just overly optimistic. Synthetic biology has also been launched with great promises that have ignited debates between actors with quite different normative foundations. Proponents of the technology have proposed to discuss its development on the basis of strict scientific risk-evaluations and this type of cost-benefit analysis has to a large extent been taken up by e.g. The Presidential Commission for the Study of Bioethical Issues who was recently asked to deliver a report on synthetic biology by Barack Obama (The Presidential Commission for the Study of Bioethical Issues 2010). On the critical side we have seen environmental NGOs, such as the ETC group, questioning the possibility of making such risk-evaluations in the face of radical uncertainty and highlighting the potential injustice in the access to potential benefits of the technology (The ETC Group 2007).

Besides the existence of these varied foundations for the debate there are also considerable differences in the ways of defining synthetic biology within the community itself and this makes it hard to draw demarcations around the practice. The different approaches, however, seem to share the ambition to utilize the technologies of human engineering to optimize evolutionary processes in biological organisms in order to make them perform specific desired functions. One of the ways this is done is by decoding the genome of an organism, translating it into digital codes on a computer, recoding it digitally and use that line of code to make a synthetic DNA-structure that can be inserted in an otherwise empty cell. This cell will then produce specific desired proteins and potentially be part of an organism that can be used to create products 
such as plants with efficient photosynthesis, algae-based biofuels and malaria-medicine (The Presidential Commission for the Study of Bioethical Issues 2010). The J. Craig Venter Institute made headlines in May 2010 by creating the world's first synthetic and self-replicatory bacterial cell and even though its genome is minimal it was by many commentators seen as a landmark of this synthetic biology. The fact that a minimal cell can create such a fuzz serves to show that the technology is still in its infancy and that synthetic biology is still a flexible technology regarding its technical development as well as the way it is interpreted by society.

\section{3: The web and synthetic biology}

The issue of synthetic biology revitalizes democratic questions about the process though which scientific technologies are discussed and stabilized. The web is one of the central arenas in which this process takes place and the flexibility in the definition and use of synthetic biology makes it an interesting case for analyzing the democratic and epistemic characteristics of the web as a space where the public can encounter different views about science (Weingart, 1998) and where diverse social institutions interact in shaping the social situation around this new technology (Hjarvard, 2008).

The importance of the web as such an arena has not escaped scholarly attention and even though the discussion has not been able to solve the disagreement between "optimists" and "pessimists" it has shown some similarities in the way the web has been approached as an object of study by both sides. A tendency has namely been to conceptualize discussions on the web as something that takes place in an online sphere (Gerhards, Schaefer 2010, Al-Saggaf 2006, van Os, Jankowski \& Vergeer 2007), in a virtual sphere (Papacharissi 2002) or in cyber-space (Marlin-Bennett 2011). It has furthermore been a common move to compare this sphere with a traditional, pre-digital sphere or, in the most explicit cases, with the "[...] real world in which people can walk around and encounter each other in the flesh” (Marlin-Bennett 2011, 9).

This theoretical move has made it necessary to decide on strategies of sampling that allows for clearly demarcated representations of the spheres in question but it has caused methodological headaches with regard to the online sphere (Lazer et al. 2009) in which it has proved to be far from obvious how to conduct a representative sample of e.g. the discussion about synthetic biology. The most well-articulated response to the challenge has been to draw the boundaries of a web-sphere on the basis of a set of web-sites that the researcher knows to be central to the topic of interest and then use them to initiate a snowball-sample through hyperlinks. A "web-sphere" is, on this account, conceptualized as "a set of dynamically defined digital resources spanning multiple web-sites deemed relevant or related to a central event, concept or theme, and often connected by hyperlinks" and the web-sphere is supposed to be representative of the discussion online (Schneider, Foot 2005). ${ }^{1}$

The merit of this way of approaching the Internet as a public sphere is that it allows for structured comparisons between spheres that are clearly demarcated, but the drawback is that it requires the researcher to collapse previously distinct entities into unified wholes. This is e.g. the case when political web-sites in EU-countries are collapsed into a representation of the "online European public sphere" (van Os, Jankowski $\&$ Vergeer 2007) or when the search result pages of Google, Yahoo and Fireball are collapsed into a representation of the "internet-based public sphere" (Gerhards, Schaefer 2010). The price for being able to do broad comparisons between the offline and the online is the reduction of complexity and distinction within these spheres.

\section{4: From spheres to web-visions}

An alternative to the focus on the representation of spheres is to take departure in the idea that every image of a controversy is a unique "socio-technical setup" produced by a specific network of actors through specific media with specific logics of filtering. This is as true for the image that ends up as the outcome of a traditional offline media of representation, such as the city-hall meeting, as it is for the image that ends up as the result of a search on Google. (Girard, Stark 2007). The way public officials facilitate a citizen hearing with the use of technologies such as a strictly planned physical space, microphones and plans for speaking and voting makes controversies visible and managable in a way that is neither more or less performative and mediated than the way Google's algorithm makes a controversy visible. Both images are produced in a situation with specific constraints on the social interaction (Hjarvard, 2008)

The interesting insights, therefore, do not lie in comparisons of how well they map pre-defined spheres but in looking at them as two distinct socio-technical set-ups that assemble and organize public experience in different ways. This situated approach to depicting controversies also seems more in line with the argument behind the concept of "ethno-epistemic assemblages", which prompts us to take our analytical point of departure in a situated setting. If knowledge-claims about synthetic biology are situated in local contexts it 
may be a fruitful move to start from the way the web looks through a specific filter, at a specific place at a specific point in time.

The move of beginning a media-analysis on the basis of the logic of the filter instead of departing from an a priori divide between different spheres also resonates with work done under the heading of mediatization-theory. With departure in the concept of "media-logics" scholars within this tradition have emphasized how the form of a given media influences the distribution of material and symbolic ressouces in a way that control the categorization, selection, circulaton and presentation of knowledge (Hjarvard, 2008). This focus on how formal and informal rules shape representations of knowledge is carried on in the study below but it is important to emphasize that these representations are not just a result of the logic of filtering and the form of the medium. Because both Google and Wikipedia rely on user-generated content their representations of synthetic biology is just as much an outcome of the behavior of all the actors that produce and rate this content. It is an assemblage of the logics of filtering, the behavior of web-users, the choices made by web-designers and the words used by the involved organizations to describe synthetic biology. The interaction between these human and non-human actors is what eventually leads to the situation where a specific scope of the dicsussion about synthetic biology becomes visible. This scope is situated in time and space and the paper will conceptualize it as the "web-vision" that the filter provides the user with. It is defined as follows:

$A$ "web-vision" is the specific actors, themes and documents that become visible to the user when querying the web through a specific information-filter at a specific time in a specific place.

This focus on spatial and temporal groundedness goes against popular conceptions of the web as a place where time and space is annihilated and this seems as a natural consequence of breaking with conceptions such as the virtual or cyberspace as something different from the offline. If these are not interesting divides it follows that we cannot think of the web as being exempt from time and space that are some of the most central conditions for any other part of our cognition.

\section{Operationalizing the "web-visions" of a British user}

In order to situate the empirical research of the paper in a spatial and temporal reality it was decided to follow the "web-visions" of entry-points to the web that are heavily used in the UK. The geographical situation in the UK was chosen for two reasons. The first is that the UK has a quite unique tradition in relation to biotechnological risk-assessment due to the fact that the BSE scandal in the 1990s made many of the abovementioned questions about science and democracy surface in the region. This led to a focus on the relevance of broad debates on scientific developments and analyzing web-visions with departure in the UK makes it possible to determine the extent to which this focus is transferred to the web as it is seen by the UK public. The second reason is that the UK shares semantics with the United States in relation to the word used to denote the scientific project we are interested in. A search for "synthetic biology", therefore, makes it possible to determine the extent to which the British public is influenced by American framings. ${ }^{2}$ The filters to follow were chosen on the basis of a search on Alexa.com, which revealed wikipedia.com and google.co.uk to be among the most heavily used entry-points to the web by British users. While inquiring these filters about the issue of synthetic biology it was ensured that all the searches were conducted from the same computer with a constant IP-address based in London and that the Google-searches were de-personalized by adding " $\mathbf{x p w s}=\mathbf{0}$ " after the search URL. In that way the search-results represents the way Google makes the controversy visible before the $(\mathrm{minor})^{3}$ influence of browser history kicks in.

Besides being heavily used the two filters were chosen because they rely on different forms of crowd-sourcing and therefore serve the potential of producing different web-visions around synthetic biology. Google originally created its position on the market of search by harnessing the words and hyperlinks that people constantly leave on the web and it used these traces to statistically calculate a rank of relevance for any web-site accessible through their interface (Brin, Page 1998). This approach to information-filtering and relevance was launched in opposition to e.g. Yahoo that relied on human-based classification of web-sites in the tradition of library indexes. Google can in that sense be said to utilize the "crowd-wisdom" (Sunstein 2006) of its users by letting e.g. hyperlinks count as votes for web-sites. It is this crowd-logic, rather than the fact that it plays out in an online realm, that makes it an interesting case to follow. Moving to Wikipedia it has, in the same sense, taken advantage of the fact that users of the web are becoming producers of web-content by enabling a transparent system of collaborative filtering of its articles. Everybody can edit an article on the encyclopedia that works on the basis of a post-filtering philosophy. This means that a contribution to an article immediately appears on the site without any form of editorial oversight. Instead of pre-defined filters, Wikipedia has an internal hierarchy of moderators and users that constantly overlooks articles and removes knowledgevandalism (Bruns 2008) in a way that seem to be effective in correcting errors (Fallis 2008). The two filters are, 
accordingly, harnessing the intelligence of web-users on the basis of different philosophies of filtering and what makes them possible to compare is that they both function as hubs for sending the user further into the web. It is true that the page of search-results of Google and the article on Wikipedia are quite different but a central part of both of them is to decide on the relevance of external links to guide their users further into the web.

It is these links that form the basis of the operationalization of the "web-vision" that the filter gives rise to. In the case of Google the web-visions are simply made by following the links of the top 20 URLs returned in the search-result and in Wikipedia they were made by following the external links in the bottom of the article. The links were followed with the help of the Issue Crawler ${ }^{4}$ that set to follow two layers of links as well as to perform a "co-link analysis", which in the language of the Issue Crawler means that only pages with two in-links are kept in the visualization. This was done in order to reduce the "web-visions" by restricting them to include only sites that are deemed relevant by at least two other web-sites and thereby reduce the risk that the visuali- zations would drift away from the issue of synthetic biology. The raw data that the Issue-Crawler returns is a matrix illustrating which web-sites that are linking to each other and this data was directly exported into UCI-net which allowed for subsequent manipulation of the networks returned from the crawler. This manipulation included the deletion of all web-sites that did not have at least one mention of the word "synthetic biology" throughout its pages as well as "irrelevant" links such as the ones that almost all web-sites forge to e.g. the licenses of Creative Common and Flash-players. ${ }^{5}$ Finally the visions were made interpretable and useful for the study by organizing the networks on the basis of statistical calculations of the distances between nodes and by coloring, shaping and sizing the nodes in the network on the basis of the parameters in the table below.

The shaping and sizing of the nodes in the "webvisions" is a deductive element in the sense that the parameters are based on already established theoretical expectations of what a controversy is. The six web-visions below are accordingly a construct on which the researcher has a huge influence. No web-users will en-

Table 1: Parameters used to shape the visualizations in UCI-net

\begin{tabular}{|c|c|c|c|}
\hline Type of parameter & Parameter & Explanation of the parameter & Visualization \\
\hline Temporal & $\begin{array}{l}\text { New web-sites in the } \\
\text { vision }\end{array}$ & $\begin{array}{l}\text { Looks at whether there are new URLS in a specific vision } \\
\text { compared to the previous month in order to detect the type } \\
\text { of fluidity there are in the visions. }\end{array}$ & $\begin{array}{l}\text { Size of nodes } \\
\text { Big node }=\text { New } \\
\text { Small node }=\text { Recurring }\end{array}$ \\
\hline Structural & $\begin{array}{l}\text { The existence of clusters } \\
\text { and brokers }\end{array}$ & $\begin{array}{l}\text { Looks at the extent to which the URLS are organized in } \\
\text { clusters, whether these clusters have specific characteristics } \\
\text { and whether there are URLLs that serve as brokers between } \\
\text { clusters. This is done in order to detect the polarization of } \\
\text { the issues and the actors capable of mediating between } \\
\text { polarized parties. }\end{array}$ & Spring-based graphs \\
\hline Spatial & $\begin{array}{l}\text { The geographical origin } \\
\text { of the visible URLS }\end{array}$ & $\begin{array}{l}\text { Looks at the geographical origin of the URLs in order to } \\
\text { geo-locate the issue. }\end{array}$ & $\begin{array}{l}\text { Shape of nodes } \\
\text { Round = US } \\
\text { Square = UK } \\
\text { Diamond = Global } \\
\text { Circle in square = Canadian } \\
\text { Triangle = Other Europe } \\
\text { Plus = Other World }\end{array}$ \\
\hline Spatial & The type of organization & $\begin{array}{l}\text { Looks at each URL in terms of the type of organization it } \\
\text { represents in order to detect the kind of actors dominating } \\
\text { the issue and the kind of actors that are connected to each } \\
\text { other. }\end{array}$ & $\begin{array}{l}\text { Color of nodes } \\
\text { Red = Policy advise, social science or } \\
\text { public engagement } \\
\text { Blue = Commercial } \\
\text { Green = Natural Science } \\
\text { Yellow = News \& Magazine } \\
\text { Purple = Funding } \\
\text { Dark Red = Governmental } \\
\text { Black = Other }\end{array}$ \\
\hline
\end{tabular}


Figure 1: Google UK - January

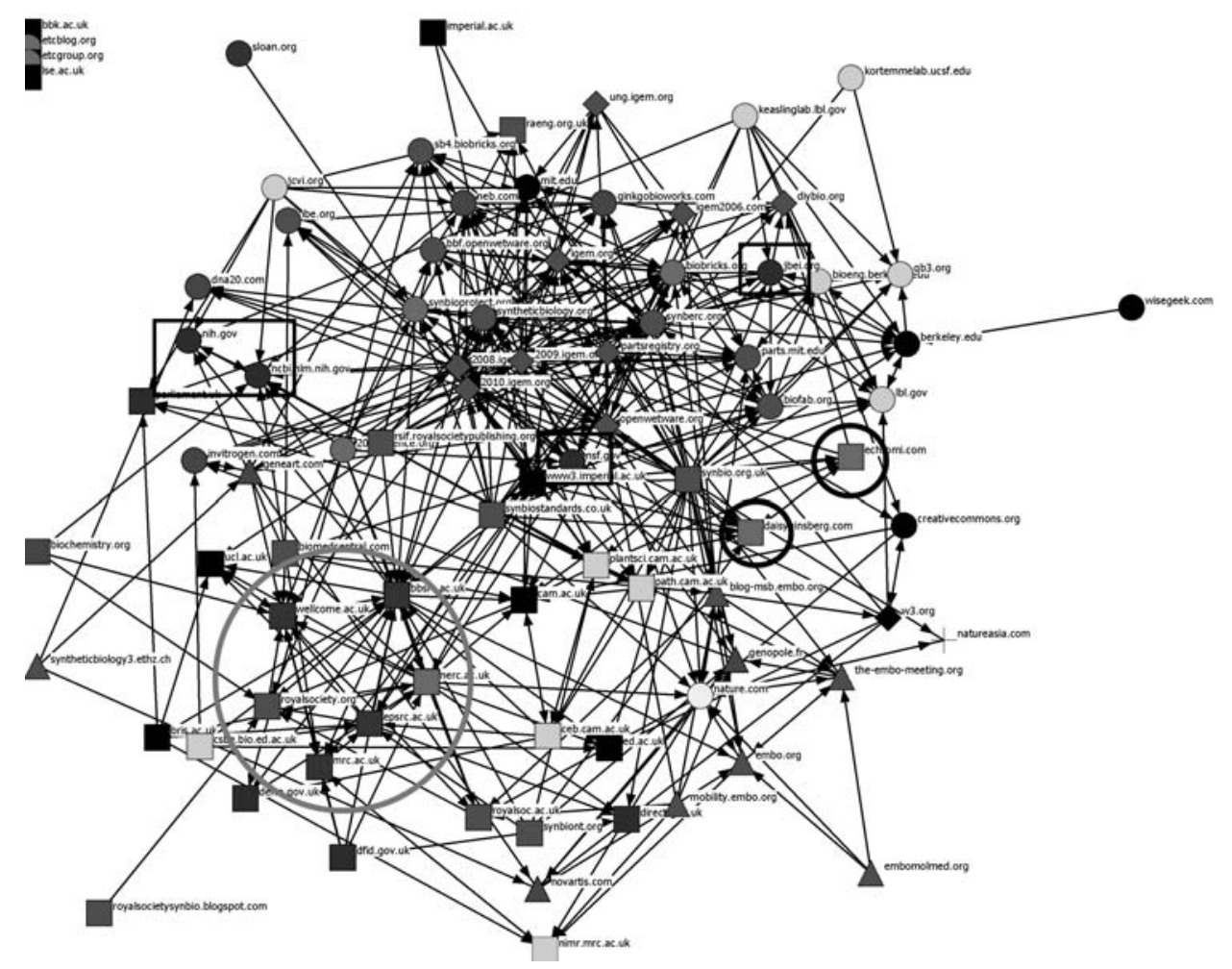

Figure 2: Google UK - April

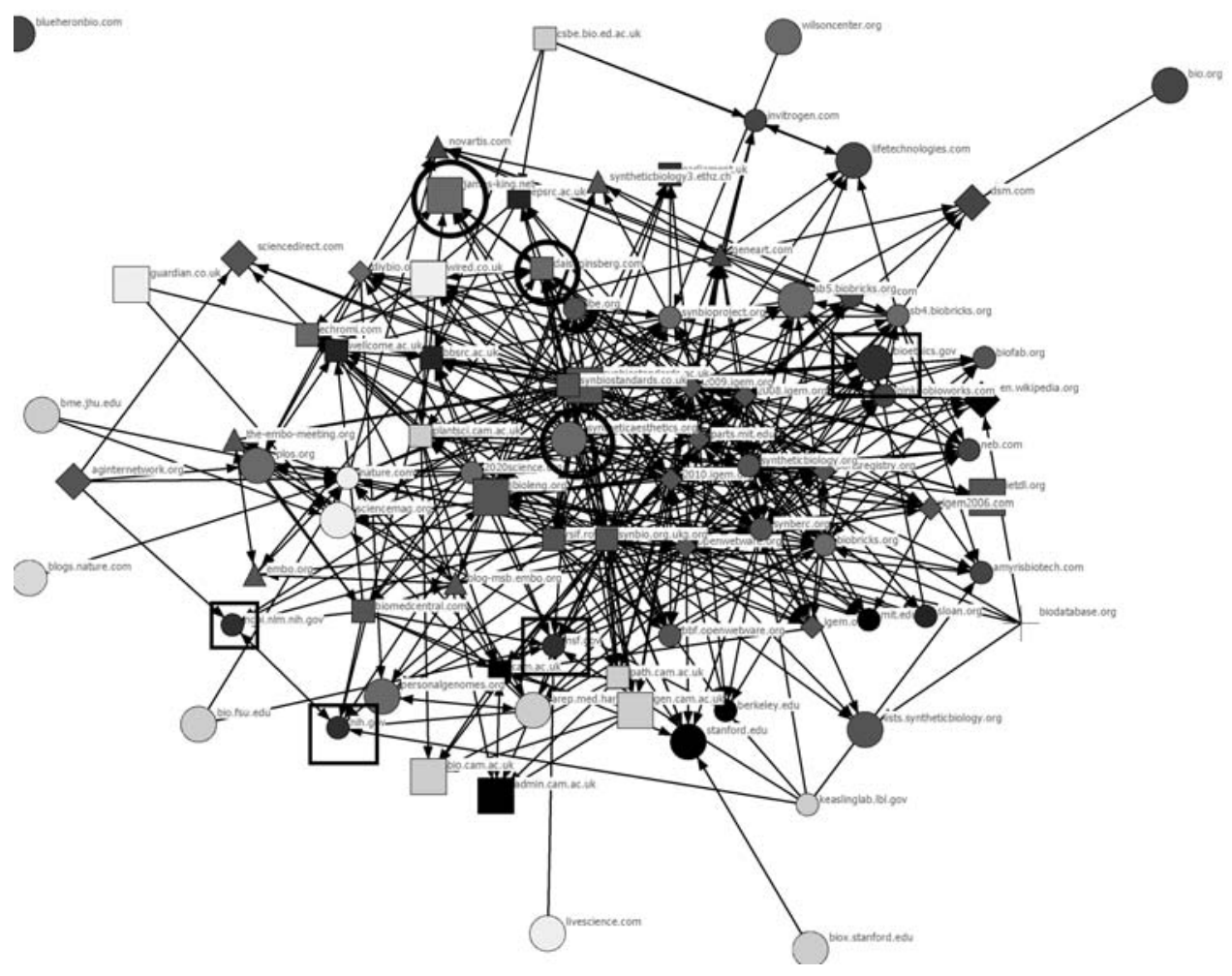


Figure 3: Google UK - June

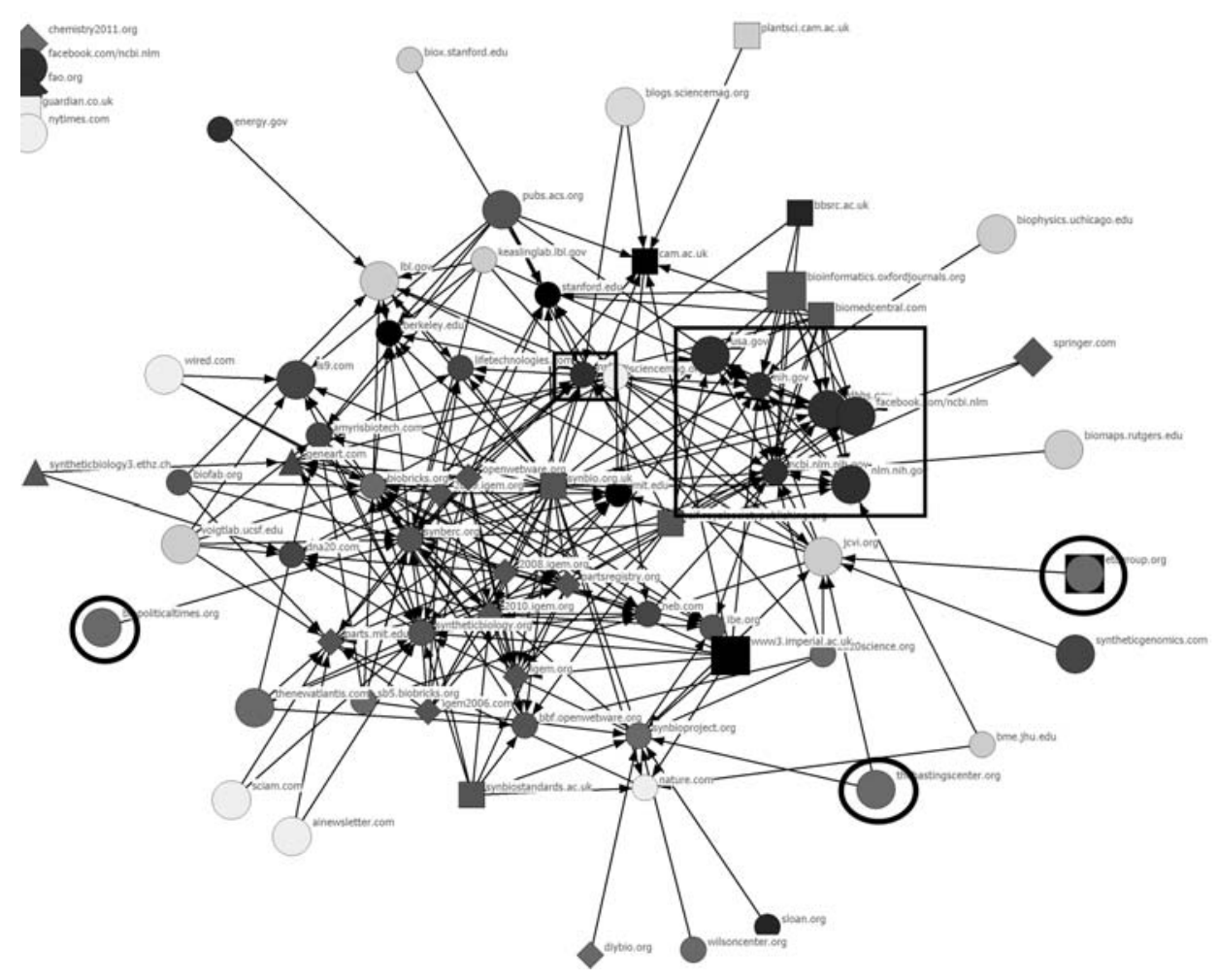

Figure 4: Wikipedia - January

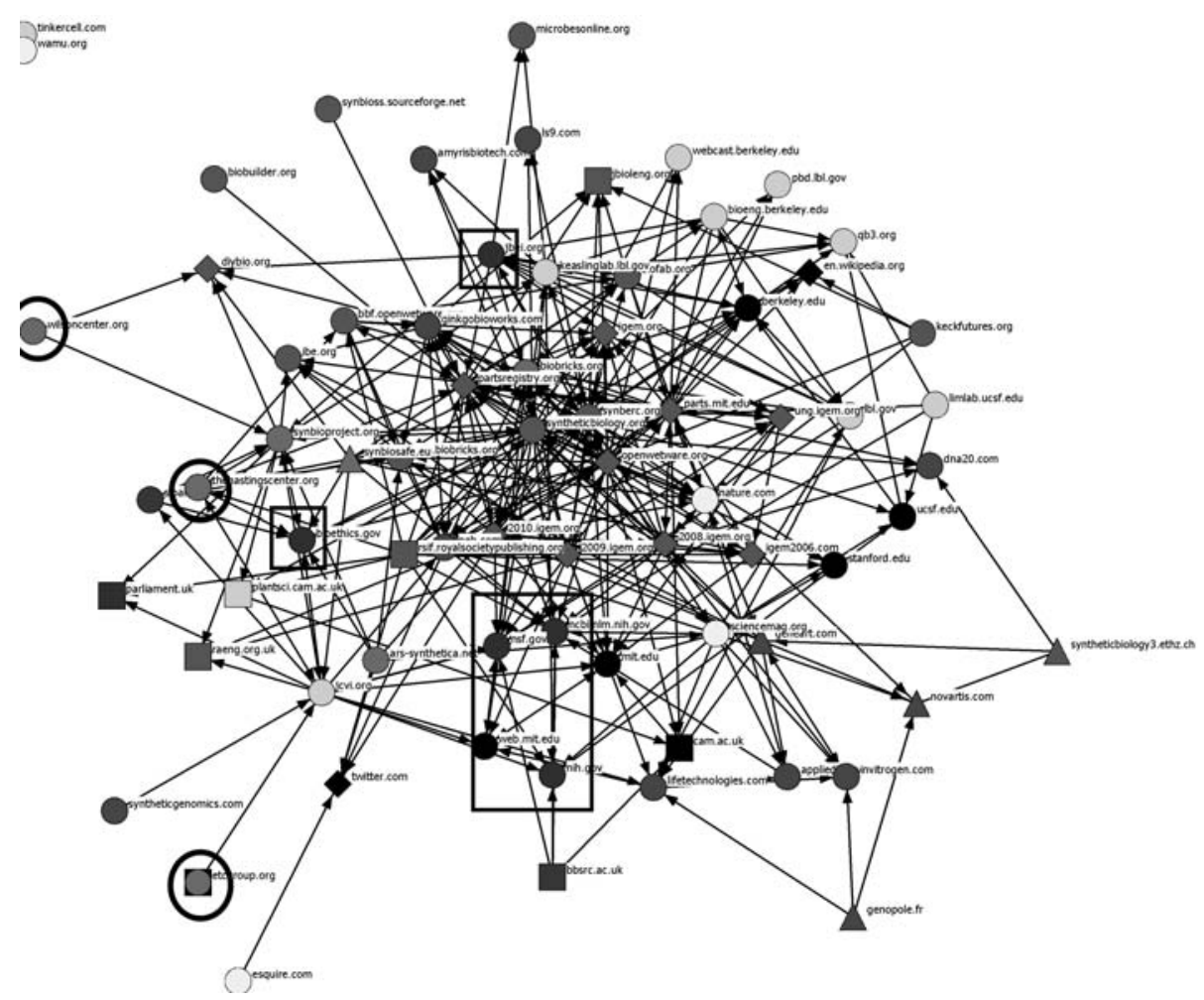


counter these visualizations when browsing the web for information about synthetic biology and they are only to be seen as heuristic images of the scope of the controversy that the chosen filters make visible to a generalized UK web-user. They are prompts for discussing what lies "behind" the URL-lists that the filters return. The next section will outline some of the most interesting findings in this initial stage of the study and thereby provide an insight into the dynamics with which the two filters demarcate the controversy of synthetic biology.

\section{5: Similarities and differences in the visions}

The temporal make-up of the public space is important and we will therefore start by looking at the extent to which the visions are stable or fluid by comparing the way they make actors visible over time. The sizing of the nodes in the web-visions is supplemented with a calculation of a "fluidity-rate" that indicates changes in the visions of a filter from month to month and a "longitudinal stabilityrate" indicating the level of stability in the visions across the whole period. They are operationalized according to the formulas below and the boxes compare the visions on these concepts.

Fluidity-rate $=$ (number of new web-sites in the web-vision prior to previous month/total amount of websites in the given month) $\mathrm{x} 100$

Logitudinal stability-factor $=(($ websites common to all visions of a single devise across $y$ months/avarage amount of web-sites in the visions across $y$ months) $\mathrm{x}$ 100).

Table 2: Tables of fluidity-rates and longitudinal stability rates

\begin{tabular}{|l|c|c|c|}
\hline Google.co.uk & Jan & Apr & June \\
\hline Number of web-sites & 86 & 83 & 73 \\
\hline Fluidity-rate & - & 45 & 38 \\
\hline Longituninal stability-rate & \multicolumn{3}{|c}{43} \\
\hline
\end{tabular}

\begin{tabular}{|l|c|c|c|}
\hline Wikipedia & Jan & Apr & June \\
\hline Number of web-sites & 73 & 80 & 87 \\
\hline Fluidity-rate & - & 19 & 28 \\
\hline Longituninal stability-rate & \multicolumn{3}{|c|}{69} \\
\hline
\end{tabular}

From the two boxes it is evident that the Google-visions are way more fluid and changing than the Wikipediavisions and this serves as an indication that the collaborative and consensus-oriented logic of filtering behind Wikipedia and the type of actors that makes up this as-
Figure 5: Google UK-visions: Actor-percentage in relation to the total amount of web-sites in the respective visions.

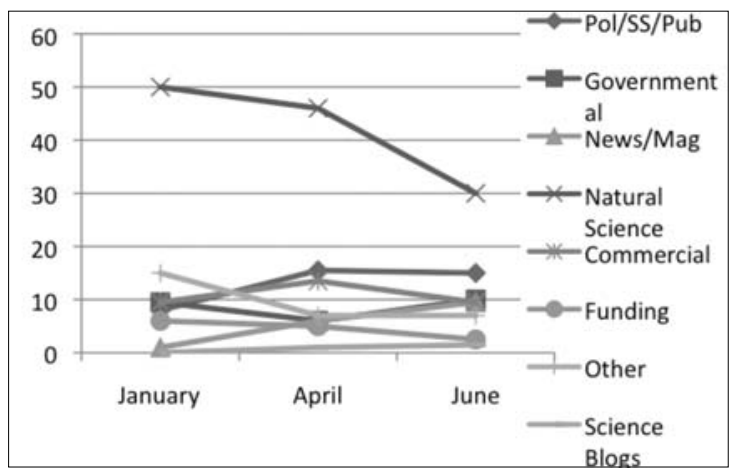

Figure 6: Google UK-visions: Geography-percentage in relation to the total amount of web-sites in the respective visions.

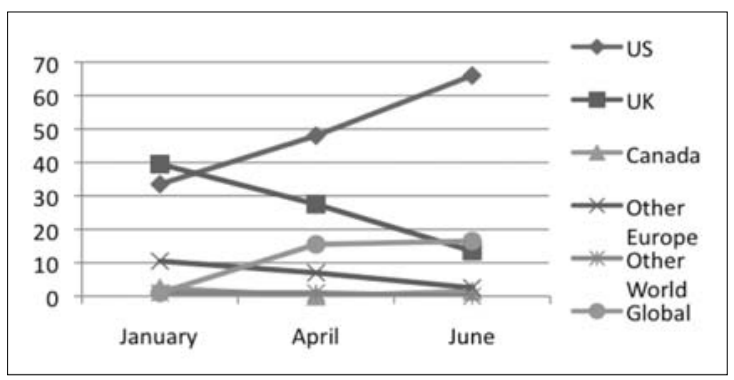

Figure 7: Wikipedia-visions: Actor-percentage in relation to the total amount of web-sites in the respective visions.

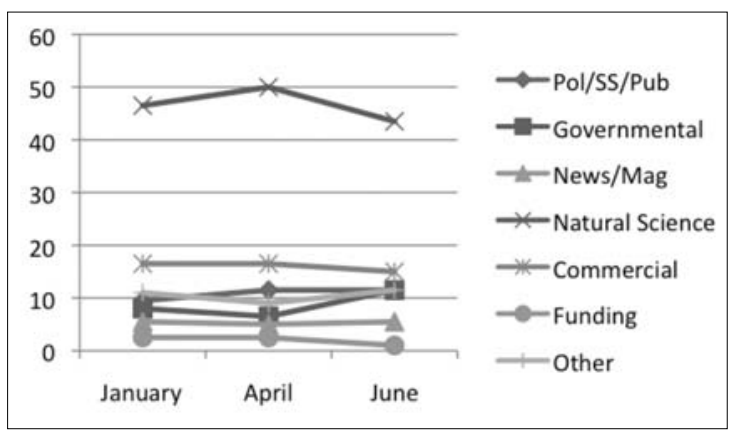

Figure 8: Wikipedia-visions: Geography-percentage in relation to the total amount of web-sites in the respective visions

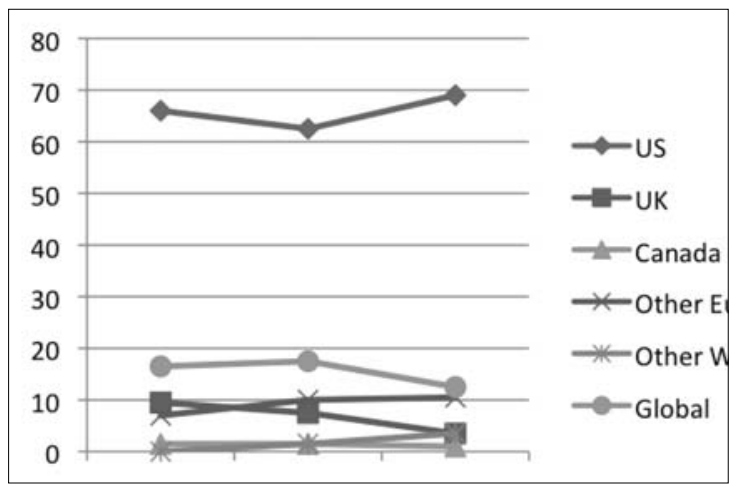


semblage results in a slower updating-pattern than the crowd-based algorithm of Google and the actors that are active here. Besides that it indicates that the Wikipediavision is cumulative in the sense that the number of links are increasing over time. Few web-sites drop out and this is also indicated by the fact that the longitudinal stabilityrate is high. In order to better understand the characteristics of these differences in temporality it is, however, necessary to look at the distribution of web-sites in the different visions over time with reference to the spatial parameters listed above.

Looking at the graphs above we can see that the high fluidity factor in the Google-visions is generally translated into less straight lines compared to the Wikipedia-visions where the lines are quite stable. This is especially evident in relation to the geographical distribution of web-sites, which is changing dramatically in the Google-visions in the time-span covered. The US actors are on the rise and a comparison of January and June reveals that a tightly connected UK-cluster consisting of e.g. BBSRC, The Natural Environmental Research Council, The Welcome Trust and The Royal Society have disappeared from the vision in during this time-span (the cluster is marked with a red circle in the January vision above). The fact that there seems to be a distinction between this UK cluster and the US cluster to the right of it makes it even more interesting that the fluidity in the Google-vision to a large extent covers an Americanization. A subsequent qualitative analysis must inquire into whether or not there are radically different narratives about synthetic biology in the disappearing cluster and the US cluster. Another finding is that the actors that are positioning themselves as being global voices are to a large extent connected to the US cluster. When you search for an organization that presents itself as having a global perspective on synthetic biology you will, accordingly, quickly find yourself browsing the websites of American organizations. Along with this Americanization, the fluidity in the Google-vision also covers a shift in the types of websites that are made visible and it is especially news actors are on the rise whereas natural science actors that are in decline. The emerging news-sites are to a large extent US-based and even UK-based sites, such as The Guardian, are running stories on American project on synthetic biology.

The characteristics of the fluidity in the Google-vision is, however, not fully understandable by looking at the graphs above. Figure 9 e.g. indicates that not much have happened regarding the red nodes in the Google-visions because they make up the same share of the network in April and June. But looking closer at the visions we can see that there is a change in this category as well. Even through the percentage of this type of web-sites is similar in April and June we can see that the web-sites that represent this group are becoming more institutionalized as well as less UK-oriented over time. UK-based designers such as Daisy Ginsberg and James King have e.g. dropped out of the vision. Their project is to illustrate social issues around synthetic biology through art and we see that their visibility is substituted by the visibility of The Hastings Center, The ETC Group and Biopolitical Times that are all more institutionalized American NGOs (all of these are marked with black circles on the visions above).

When looking at the fluidity of Google from the perspective of the Wikipedia-visions we can see that the June-vision of the filter is similar to the January version of the Wikipedia-vision which haven't changed much from January to June. This is true when we look at the graphs in Figures 5-8 as well as when we look at the more detailed network-visualizations in Figures 1-4. The Google-visions had a unique scope of visibility in the first months with more UK web-sites and a less institutionalized set of social actors being visible, but the different logics of filtering seem to be generating increasingly similar visions. This may be due to the fact that The Presidential Commission for the Study of Bioethical Issues released a report on synthetic biology in late December and this report is becoming visible in the Google.co.uk-visions in April where it is linked to by a group of centrally positioned actors. These links are not visible in the June-visions of Google.co.uk, but a range of American institutions have become visible instead. Looking at the Wikipedia-visions we see that these institutions have been visible from the outset and this is one of the significant examples of the increasing similarity across the two filters (these institutions are marked with black squares in all visions above). If the publishing of the American report is the explanation for the increasing similarity it serves to show that the Google-vision is easily influenced by such big events and that they have the power to trump the visibility of the actors that made these visions stand out from the Wikipedia-visions in January. The Wikipedia-visions, on the other hand, has a high "logitudinal stability-rate" and the visions are not altered dramatically by the links forged to the Obama-report. Possibly because they fit the already institutionalized slant of the vision.

\section{Making things public?}

These quantitative analyses represent the beginning of longitudinal study but they already serve to indicate answers to the important question of what visualizations of web-visions could potentially be useful for. One possible answer is that they make the structure of controversies public in a way that allows people to better understand and navigate these controversies. But we need to remem- 
ber that the web is not the world and accept that if visualizations are to serve the function of a good public map they need to be constructed in a way such that many more layers of information is added to the digital traces of one filter (Venturini, 2010). It would therefore be too ambitious to present the "web-visions" above as tools for navigating the controversy of synthetic biology. Quite to the contrary they are constructed by following specific filters in a specific country at a specific time and they are even removed from our everyday experience of the web through the use of the issue-crawler. They are, in other words, a construct make by several actors - including the researcher.

But the lack of representation is at the same time the roots of their usefulness in at least three ways. From the perspective of this paper they are, first of all, useful because they highlight the fact that it is filtering logics and the assemblages they are part of, rather than ontological distinctions between the offline and the online, that is important when we try to understand the dynamics of controversies and their mediation. And even though they lack the quality of representation they may still provide insights into the dynamics of controversies. Working on the basis of case-study logic one could e.g. argue that the Wikipedia-visions are "least likely" to change radically and that rapid fluctuations in this vision would indicate a very dynamic controversy whereas changes in the Google UK visions are less remarkable. This just serves to indicate that representativity and objectivity is not the only benchmark of good visualizations and deeper discussions of the methodological underpinnings of controversy-visualizations can be found in another paper (Koed Madsen, 2012).

Secondly, the fact that the visions are situated in a UK-context makes them interesting cases for studying the development of biotechnological discussions in the specific filters that are used by the public in a country that has a unique history regarding such discussions. The fact that the vision of these filters seem to be Americanized and institutionalized after the release of the Obamareport raises interesting questions about the role digital medias play in the UK. Is it e.g. a problem for the UK public that they share language with The United States when it comes to searches? Is it a problem that the UK does not have a national Wikipedia like other countries? In order to probe further into such questions it could e.g. be interesting to compare the UK findings with webvisions that are situated in other countries.

The third answer to the question of relevance is broader and it points to the fact that these visions are assemblages that are influenced by a range of different phenomena such as algorithms and information-filters, cultures of relevance, cultures of media use and - most importantly - digital traces left by the organizations involved in the controversy of synthetic biology. For a researcher they represent a way to visualize and simplify controversies in a way that takes departure in the current cultures of communication and the vocabulary of the actors engaged in the controversies (Latour 2007). This makes them quite different from other simplifications of controversies such as surveys and city hall meetings. The dynamics behind their creation makes potential differences interesting as reflections on e.g. media-logics and organizational networks. Compared to an analysis of e.g. the search-page that meets the everyday web-user on Google, these visions make explicit the networked nature of influential web-based filters.

\section{6: Conclusion and further research}

The empirical analysis above have presented a longitudinal comparison of web-visions created by two influential web-filters that sort information according to quite different logics of relevance. The analysis of these visions serves as an empirical example of the way the web is better understood as a conglomerate of filters that make visions available to its users rather than as a unified sphere that can be representatively uncovered. The comparison between the visions produced by google.co.uk and wikipedia.com revealed differences in the temporality of the visions. The Google-visions proved to be altered significantly over time whereas the Wikipedia-visions remained stable. The analysis showed that the fluidity in the Google-visions was correlated with rising discussions about a report released by The Presidential Commission for the Study of Bioethical Issues in the USA and that e.g. the visibility of artists posing social questions about the technology were declining. The discussion following the analysis provided three arguments for the usefulness of "web-vision" analysis that hinted at the potential of using them to say something more general about the world by constructing the visions on the basis of a case-study logic rather than on the logic of sampling spheres, their potential to help analysis of geographically and temporally situated developments of the web as a public arena and their potential to provide insights in to the assemblages and dynamics behind the simplified outcomes of the filters.

The results represent the beginning of a longitudinal study and further research in the area needs to see the quantitative studies in this paper being supplemented with qualitative studies of the themes and connections between the actors in order to generate a deeper understanding of the differences between the demarcations that different filters give rise to. They also need to be supplemented with searches for other issues in order to see 
if some of the findings above have a more general nature and with words that are related to synthetic biology in order to test the power of the search-word used. Would searches for synthetic life e.g. provide radically different visions? Finally it would strengthen our understanding of the web as a public arena if we were to conduct similar studies on search engines specifically oriented towards blogs. This would also require analyses of why people are leaving digital traces in different media-spaces and thereby give the user a larger role in the analysis. Besides these shortcomings the paper has hopefully given a sense of the kind of insights that a web-vision analysis can provide as to how the web is active in creating "visions" of controversies.

\section{References}

Al-Saggaf, Y. 2006, "The online public sphere in the Arab world: The war in Iraq on the Al Arablya website", Journal of Computer-Mediated Communication, vol. 12, no. 1, pp. 311-334.

Benkler, Y. 2006, The wealth of networks - How social production transforms markets and freedom, Yale University Press, London.

Brin, S. \& Page, L. 1998, "The anatomy of a large-scale hypertextual Web search engine”, Computer Networks and ISDN Systems, vol. 30, no. 1-7, pp. 107-117.

Bruns, A. 2008, Blogs, Wikipedia, Second life, and beyond: From production to produsage, Peter Lang, New York, NY.

Coleman, S. 2005, "New mediation and direct representation: reconceptualizing representation in the digital age”, New Media \& Society, vol. 7, no. 2, pp. 177-198.

Fallis, D. 2008, “Toward an epistemology of Wikipedia", Journal of the American Society for Information Science and Technology, vol. 59, no. 10, pp. 1582-1597.

Gerhards, J. \& Schaefer, M.S. 2010, "Is the internet a better public sphere? Comparing old and new media in the USA and Germany", New Media \& Society, vol. 12, no. 1, pp. 143-160.

Girard, M. \& Stark, D. 2007, "Socio-Technologies of Assembly: Sense Making and Demonstration in Rebuilding Lower Manhattan" in Governance and information technology From electronic government to information government, eds. V. Mayer-Schönberger \& D. Lazer, MIT Press, Cambridge, MA, pp. 145-177.

Goldman, A. 2008, "The Social epistemology of Blogging" in Information Technology and Moral Philosophy, ed. J.V. Hoven, Cambridge University Press, pp. 111-123.

Halavais, A. 2009, Search Engine Society, Polity Press, Cambridge, UK. Hjarvard, S. 2008, "The Mediatization of Society - A Theory of the Media as Agents of Social and Cultural Change", Nordicom Review, vol. 29, no. 2, pp. 105-134.

Irwin, A \& Michael, M. 2003, Science, Social Theory and Public Knowledge, Milton Keynes, OPen University Press.

Jasanoff, S. 2007, Designs on Nature: Science and Democracy in Europe and United States, Princeton University Press, New Jersey.

Koed Madsen, 2012 (forthcoming), "Web-visions as controversy-lenses", Interdisciplinary Science Review, 37 (1).
Latour, B. 1987, Science in Action - How to follow scientists and engineers through society, Harvard University Press, Cambridge, Massachusetts

Latour, B. 2007, Beware, your imagination leaves digital traces, Times Higher Literary Supplement.

Lazer, D., Pentland, A., Adamic, L., Aral, S., Barabasi, A., Brewer, D., Christakis, N., Contractor, N., Fowler, J., Gutmann, M., Jebara, T., King, G., Macy, M., Roy, D. \& Van Alstyne, M. 2009, "SOCIAL SCIENCE: Computational Social Science”, Science, vol. 323, no. 5915, pp. 721-723.

Marlin-Bennett, R. 2011, "I Hear America Tweeting and Other Themes for a Virtual Polis: Rethinking Democracy in the Global InfoTech Age", Journal of Information Technology \& Politics, vol. 8.

O'Reilly, T. 2006, The Architecture of Participation. Available at http:// oreilly.com/pub/a/oreilly/tim/articles/architecture_of_participation.html

Papacharissi, Z. 2002, "The virtual sphere”, New Media \& Society, vol. 4, no. 1, pp. 9-27.

Rittel, H.W.J. \& Webber, M.M. 1973, "Dilemmas in a General Theory of Planning”, Policy Sciences, vol. 4, no. 2, pp. 155-169.

Rogers, R. 2009, The end of the virtual: digital methods, Vossiuspers UvA.

Schneider, S.M. \& Foot, K.A. 2005, "Web sphere analysis: An approach to studying online action" in Virtual Methods - Issues in social research on the internet, ed. C. Hine, Berg, Oxford, pp. 157-171.

Sunstein, C.R. 2006, Infotopia - How many minds produce knowledge, Oxford University Press, New York.

Surowiecki, J. 2004, The wisdom of crowds, Anchor Books, New York.

The ETC Group 2007, Extreme Genetic Engineering - An introduction to synthetic biology, The ETC Group, Montreal.

The Presidential Commission for the Study of Bioethical Issues 2010, New Directions: The ethics of synthetic biology and emerging technologies, The Presidential Commission for the Study of Bioethical Issues in the USA, Washington D.C.

Thelwall, M. 2009, Introduction to webometrics: Quantitative web reserach for the social sciences, Morgan \& Claypool.

van Os, R., Jankowski, N.W. \& Vergeer, M. 2007, "Political communication about Europe on the Internet during the 2004 European Parliament election campaign in nine EU member states", European Societies, vol. 9, no. 5, pp. 755-775.

Venturini, T. 2010, "Building on faults: how to represent controversies with digital methods", Public Understanding of Science.

Wynne, B. 1992, "Misunderstood misunderstanding: social identities and public uptake of science", Public Understanding of Science, vol. 1, no. 3, pp. 281-304.

\section{Noter}

1 A way of softening the divide between the offline and the online as well as putting less emphasis on a priori selected actors has been to distinguish between spheres within the confines of the Internet, such as the blogosphere and the news-sphere and let "ordering devises" such as BlogPulse and Google News draw the boundaries around these spheres (Rogers 2009). This is a way of letting the "logics of the web" draw the boundaries instead of snow-balling from a priori known actors. It grounds the representation in online dynamics, but it retains the ambition of uncovering a sphere.

2 The keyword searched for ended up being "synthetic biology" because searches on Google Trends, Blog-Pulse and the history of Wikipedia proved that there is much more web-activity around this key-word than around similar words such as "synthetic life", "constructive biology" or the abbreviation "synbio". 
3 In order to test whether personalization of search has effects that are so huge that the research design of the paper could be called into question, I sat up an experiment in which people from different countries conducted the same search for "synthetic biology" on their respective computers. The result of the study indicated that personalization may change a few rankings, but since it is mostly in the lower end of the top 20 it did not seem to have a significant enough impact to challenge the research design presented here.

4 www.issuecrawler.net

5 If the links to Creative Commons, on the other hand, had to do with the issue of e.g "open source biology" they were kept in the visualization. 